\title{
HOXB13 regulates the prostate-derived Ets factor: Implications for prostate cancer cell invasion
}

\author{
IN-JE KIM $^{1 *}$, TAEK WON KANG $^{2 *}$, TAEOH JEONG $^{1}$, YOUNG-RANG KIM $^{1}$ and CHAEYONG JUNG ${ }^{1}$ \\ Departments of ${ }^{1}$ Anatomy and ${ }^{2}$ Urology, Chonnam National University Medical School, Gwangju 501-746, Republic of Korea
}

Received February 26, 2014; Accepted April 29, 2014

DOI: $10.3892 / \mathrm{ijo} .2014 .2485$

\begin{abstract}
HOXB13 has been shown to enhance the invasive potential of breast and endometrial tumors. HOXB13 is also abundant in castration-resistant prostate tumors. To determine the invasive potential of HOXB13 in prostate tumors, highly metastatic PC3 prostate cancer cells were manipulated to express HOXB13 and/or the prostate-derived Ets factor (PDEF). The PDEF is believed to reduce the invasive potential of various tumors, including prostate tumors. To further demonstrate the functional correlation between HOXB13 and PDEF, transwell invasion and gelatin zymography assays were performed. In addition, the western blot analysis was used to demonstrate the expression of PDEF target proteins involved in cancer cell migration and invasion, MMP-9 and survivin. According to the results, HOXB13 promoted PC3 cell migration and invasion. The DNA microarray analysis demonstrated that HOXB13 significantly suppressed the expression of the PDEF. Accordingly, the expression of MMP-9 and survivin was regulated by HOXB13. In addition, HOXB13 promoted the invasive potential of PC3 cells while inhibiting the PDEF. The coexpression of HOXB13 and the PDEF led to moderate retardation of the number of invasive cells, indicating that HOXB13 functionally counteracted cell invasion by reducing PDEF expression. The western blot analysis demonstrated that HOXB13 counteracted the PDEF-mediated inhibition of the expression of PDEF target proteins such as MMP-9 and survivin. The results suggest that the HOXB13-mediated promotion of tumor cell invasion is accomplished mainly through the downregulation of PDEF expression.
\end{abstract}

\section{Introduction}

HOXB13 is a transcription factor with a homedomain. Although the expression of HOXB13 is limited to the normal

Correspondence to: Professor Chaeyong Jung, Department of Anatomy, Chonnam National University Medical School, 160 Baekseo-ro, Dong-gu, Gwangju 501-746, Republic of Korea

E-mail: chjung@jnu.ac.kr

*Contributed equally

Key words: HOXB13, PDEF, Ets, MMP-9, survivin, prostate cancer prostate and rectum (1), the altered regulation of HOXB13 expression has been demonstrated in various cancer tissues originating from the prostate, breast and ovary (2-4). HOXB13 controls cancer cell proliferation, but its role varies according to the type of tissue and the cancer cell environment (5-8). In addition, HOXB13 has been shown to be overexpressed in castration-resistant prostate cancer cells (9), suggesting that HOXB13 may play a role in prostate cancer metastasis. In fact, HOXB13 has recently been demonstrated to promote prostate cancer invasion by reducing the level of intracellular zinc (10).

The prostate-derived Ets factor (PDEF) is a new member of the Ets transcription factor. The PDEF is also known as a sam-pointed domain containing the Ets transcription factor (SPDEF). The human Ets family includes 27 members, and the mouse Ets family, 26 members. The DNA-binding domain has 85 amino acids that bind to DNA sequences with the GGA(A/T) core consensus sequence (11). These Ets transcription factors regulate target gene expression through cooperation with other transcription factors and co-factors and play an important role in a number of cellular processes, including the regulation of cell differentiation, proliferation, angiogenesis, and apoptosis. In addition, Ets family members play major roles in tumorigenesis, particularly in prostate cancer (12-14). They are aberrantly expressed in specific cases of leukemia and major solid tumors, including breast and prostate tumors.

The Ets domain of the PDEF preferentially binds to the GGAT sequence, not to the GGAA sequence preferred by other ETS proteins (15). Because of the complexity of binding partners and regulatory mechanisms, the PDEF has been found to play many roles in various pathophysiological conditions. Most PDEF target genes are deregulated in the tumorigenic process and include the prostate-specific antigen (PSA), survivin, MMP-7, MMP-9, the urokinase-type plasminogen activator (uPA), maspin, LASP1, VASP, p21 and Slug.

The PDEF has been shown to regulate processes involved in prostate tumor cell motility and invasion and is lost during tumor progression and increases in MMP-9 levels, thereby promoting tumor cell invasion (16). In addition, the siRNA-mediated reduction of the PDEF induces an EMT-like phenotype such that mesenchymal markers increase to reduce cell adhesion and increase cell migration and invasion (17). The PDEF reduces Slug, a protein required for the EMT (18). By contrast, PDEF overexpression in PC3 prostate tumor cells influences FAK activity and cell morphology, reducing cell migration and invasion (16). This clearly suggests that 
the PDEF affects multiple pathways, as in the case of many transcription factors, and that separating the pathways directly regulated by the PDEF from those indirectly regulated is critical to understanding the functional role of the PDEF.

This study provides a better understanding of the role of HOXB13 in prostate cancer metastasis and the mechanisms by which HOXB13 functions. Because of the highly controversial function with a very limited understanding of the regulation of PDEF expression, this study also clarifies the invasive role of the PDEF in highly malignant PC3 prostate cancer cells. Finally, we report on the regulatory mechanism underlying PDEF expression.

\section{Materials and methods}

Cell culture. LNCaP and PC3 human prostate cancer cell lines were routinely cultured in RPMI media (Life Technology, Grand Island, NY, USA) supplemented with 5\% FBS at $37^{\circ} \mathrm{C}$ in an atmosphere containing $5 \% \mathrm{CO}_{2}$. All cultures were fed with fresh media every 3-4 days.

Plasmids and reagents. pCDNA-HOXB13 was constructed from the pFLAG-HOXB13 backbone to accommodate the neomycin-resistant gene. pCDNA6-myc-His.c-PDEF was generously provided by Dr C.J. Bieberich of the University of Maryland, Baltimore, MD. The selection of HOXB13-positive and PDEF-positive PC3 cells was done with neomycin (G418 sulfate; Promega Corp., Madison, WI, USA) and blastcidine S hydrochloride (Sigma-Aldrich Corp., St. Louis, MO, USA), respectively. Antibodies were purchased as follows: PDEF (Santa Cruz Biotechnology, Santa Cruz, CA, USA), c-myc (BD Biosciences, Franklin Lakes, NJ, USA), MMP-9 (Merck Millipore, Billerica, MA, USA), survivin (Santa Cruz Biotechnology) and $\beta$-actin (Sigma-Aldrich Corp.).

Stable transfection. Cells were grown to $50 \%$ confluence in P60 culture dishes $16 \mathrm{~h}$ before transfection. Transfection was carried out using Lipofectamine 2000 (Life Technology) with either the pCDNA3.1-HOXB13 or pCDNA6-myc-His.c-PDEF plasmid based on the manufacturer's protocol. After a $6 \mathrm{~h}$ transfection period, the cells were washed and fed with a medium containing 5\% FBS. At $24 \mathrm{~h}$ after transfection, G418 $(200 \mu \mathrm{g} / \mathrm{ml})$ or blasticidin $(2 \mu \mathrm{g} / \mathrm{ml})$ was added. The selection process continued with the medium changed every 3 days, and a continuous expansion was made under sufficient antibiotics (100 $\mu \mathrm{g} / \mathrm{ml} \mathrm{G} 418$ and $1 \mu \mathrm{g} / \mathrm{ml}$ blasticidine). HOXB13 transfection was also made in PDEF-expressing PC3 cells.

Western blot assay. Cells were grown to $80 \%$ confluence in P100 culture dishes containing 5\% FBS-RPMI media. Then the cells were lysed in protein extraction RIPA buffer $(25 \mathrm{mM}$ Tris-HCl pH 7.6, 150 mM NaCl, 1\% NP-40, 1\% sodium deoxycholate, $0.1 \%$ SDS) containing a cocktail of protease inhibitors (Sigma-Aldrich Corp.). Lysates were incubated for $30 \mathrm{~min}$ on ice, followed by centrifugation for $10 \mathrm{~min}$. Supernatants were measured for the protein concentration by using the Bradford assay reagent (Bio-Rad Laboratories, Hercules, CA, USA). Each cell lysate was loaded onto $10 \%$ Bis-Tris gel (Life Technology) and separated using a Bio-Rad electroporation system. After the proteins were transferred to the PVDF membrane, primary antibodies were applied (PDEF; 1-200, HOXB13; 1-100, myc; 1-500, MMP-9; 1-1,000, survivin; $1-100$, $\beta$-actin; $1-2,000$ ), followed by incubation with horse peroxidase-conjugated secondary antibodies. Enhanced chemiluminescence was used to visualize bands by LAS 3000 (Fujifilm, Tokyo, Japan).

$R T$-PCR. Cellular RNA from cells using the TRIzol reagent and its quantity were determined spectrophotometrically. Here $1 \mu \mathrm{g}$ of extracted RNA with $100 \mathrm{pM} \mathrm{d}(\mathrm{T})_{20}$ was preheated at $72^{\circ} \mathrm{C}$ for $10 \mathrm{~min}$ and reverse-transcribed with M-MLV (Promega Corp.) at $42^{\circ} \mathrm{C}$ for $1 \mathrm{~h}$. PCR was performed in a $20 \mu \mathrm{l}$ solution containing $4 \mu \mathrm{l}$ of $10 \mathrm{X}$ PCR buffer $(200 \mathrm{mM}$ Tris- $\mathrm{HCl} \mathrm{pH} 8.4,500 \mathrm{mM} \mathrm{KCl}), 0.2 \mathrm{mM}$ dNTP mixture, $1 \mu \mathrm{l}$ template cDNA and $0.2 \mu \mathrm{M}$ primers. The primer sequences were as follows: PDEF fw, 5'-ccgggtctgagcagcgtatc-3'; PDEF rv, 5'-tgctcaggctcctcaggtgg-3'; HOXB13 fw, 5'-gcctct gtccttggtgatgaac-3'; HOXB13 rv, 5'-aggccgccatccaggaaaag-3'; $\beta$-actin fw, 5'-gcaccacaccttctacaatgagc-3'; and $\beta$-actin rv, 5'-tagcacagcctggatagcaacg-3'. Amplification reactions were performed using a DNA thermal cycler (Eppendorf, Hamburg, Germany). PCR product was separated by $1 \%$ agarose gel electrophoresis.

Wound-healing assay. For the measurement of cell migration during wound healing, cells were seeded onto 6-well plates and allowed to grow to confluence. The confluent cell monolayer was wounded by pressing a sterile $100 \mu \mathrm{l}$ pipette tip down onto the plate to cut the cell sheet and mark the plate with a sharp and visible demarcation at the wound edge. The medium and debris were aspirated away and replaced by a fresh serum-free medium, and cells were incubated for $24 \mathrm{~h}$ at $37^{\circ} \mathrm{C}$. The cells were photographed every $24 \mathrm{~h}$ after wounding by phase contrast microscopy. For the evaluation of the 'wound closure', five randomly selected points along each wound were marked and the horizontal distance of migrating cells from the initial wound was measured. This assay was imaged using a microscope (Leica Microsystems, Wetzlar, Germany). All presented data are from at least three independent experiments performed in duplicate.

Matrigel invasion assay. The transwell chamber (Corning Inc., Corning, NY, USA) was coated with a thin layer of Matrigel (BD Biosciences). Matrigel was diluted in serumfree media. The $100 \mu \mathrm{l}$ of gel suspension was added to the upper chamber and incubated for $1 \mathrm{~h}$ at RT. Then the Matrigel solution was aspirated and washed once with $100 \mu \mathrm{l}$ of serum-free media. The upper chamber was placed in the bottom chamber containing $400 \mu \mathrm{l}$ of media with fibronectin $(20 \mu \mathrm{g} / \mathrm{ml})$. Cells $\left(1 \times 10^{5}\right)$ were added to the upper chamber in $300 \mu \mathrm{l}$ of serum-free media. After incubation for $24 \mathrm{~h}$ at $37^{\circ} \mathrm{C}$, cells inside the inserts were removed with cotton tips, and the invaded cells were stained by Diff Quick (Sysmex Corp., Kobe, Japan) and analyzed using a microscope (Leica Microsystems).

Gelatin zymography. PC3 cells were cultured for $24 \mathrm{~h}$ and the supernatant was collected. The samples were analyzed using a zymographic technique based on $10 \%$ SDS-PAGE with $0.1 \%$ (w/v) gelatin (Sigma-Aldrich Corp.) as the substrate. The samples were loaded without heating with sample buffer 
A

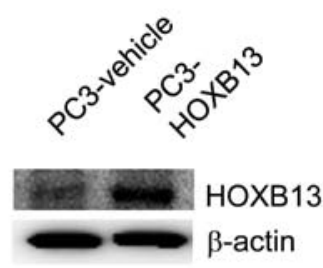

B
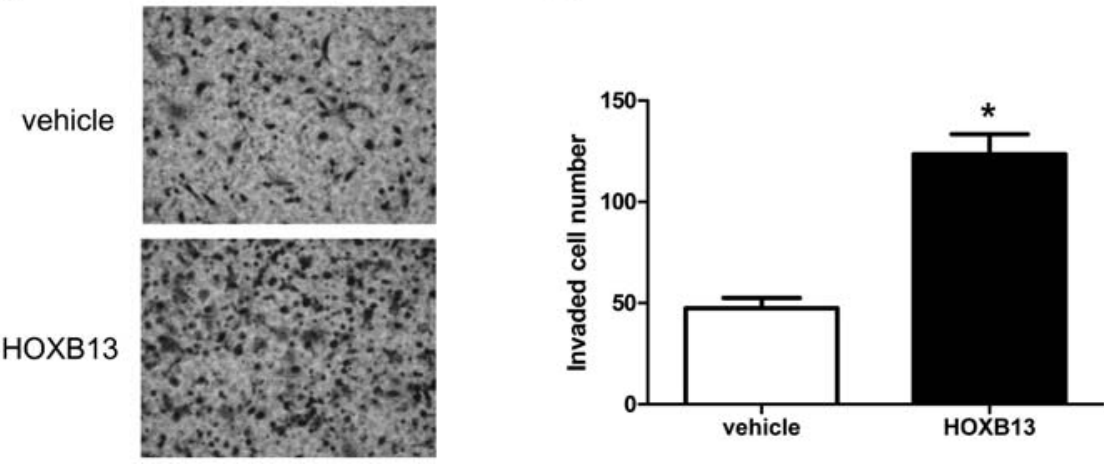

Figure 1. HOXB13 promotes the invasive potential of PC3 prostate cancer cells. (A) PC3 cells were stably transfected with either pCDNA3.1 (vehicle) or pCDNA-HOXB13 (HOXB13). (B) Cells were applied to the transwell to verify the invasiveness of each cell line. Cells are shown after Diffquick staining. (C) Invaded cells were counted on five randomly selected fields (20X) and shown as a bar graph. Student's t-test ( $\mathrm{p}<0.05)$.

and applied to a non-denaturing $10 \%$ polyacrylamide gel containing $1 \mathrm{mg} / \mathrm{ml}$ gelatin. After electrophoresis, the gel was washed twice in $50 \mathrm{mM}$ Tris $(\mathrm{pH} 7.4)$ containing $2.5 \%(\mathrm{v} / \mathrm{v})$ Triton X-100 for $1 \mathrm{~h}$, followed by two 10 min rinses in $50 \mathrm{mM}$ Tris (pH 7.4). After SDS removal, the gel was incubated overnight in $50 \mathrm{mM}$ Tris ( $\mathrm{pH} 7.5$ ) containing $10 \mathrm{mM} \mathrm{CaCl}_{2}, 0.15 \mathrm{M}$ $\mathrm{NaCl}, 0.1 \%$ (v/v) Triton $\mathrm{X}-100$, and $0.02 \%$ sodium azide at $37^{\circ} \mathrm{C}$ under constant and gentle shaking. After incubation, the gel was stained with $0.25 \%$ Coomassie brilliant blue R-250 and destained in $7.5 \%$ acetic acid with $20 \%$ methanol. Gelatinase bands appeared white on a blue background. The activity of MMP-9 was semi-quantitatively determined by densitometry.

\section{Results}

HOXB13 promotes the invasive and migratory potential of PC3 prostate cancer cells. To verify the involvement of HOXB13 in prostate cancer cell migration and invasion, PC3 cells were stably transfected with either pCDNA3.1 (vehicle) or pCDNA-HOXB13 (Fig. 1A). Selected cells were used for Matrigel-coated transwell invasion assays. As shown in Fig. 1B, there were visibly more infiltrated cells in HOXB13transfected cells. Several random fields were selected, and infiltrated cells were counted (shown as a bar graph). HOXB13 significantly increased the invasion of PC3 cells through the Martigel-coated transwell (vehicle 47.40 \pm 5.13 ; HOXB13

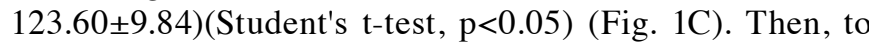
determine the involvement of HOXB13 in the migration of PC3 cells, an in vitro wound-healing assay was performed using HOXB13-manipulated PC3 cells. As shown in Fig. 2A, HOXB13 stimulated the migration of PC3 prostate cancer cells in a time-dependent manner. The bar graph representation of the migratory property shows the same result (Fig. 2B).
At $24 \mathrm{~h}$, the gap for the wounded region was 2.90 with control cells, whereas it was $1.03 \pm 0.01$ with HOXB13-transfected cells $(\mathrm{p}<0.05)$. At $48 \mathrm{~h}$, the gap for the wounded region was $2.20 \pm 0.10$ with control cells, whereas it was $0.55 \pm 0.07$ with HOXB13-transfected cells $(\mathrm{p}<0.05)$.

HOXB13 manipulates the expression of genes involved in prostate cancer cell migration and adhesion. To profile HOXB13 target genes, LNCaP prostate cancer cells were infected with either adenoviral HOXB13 or an empty vehicle, followed by a cDNA microarray analysis, as demonstrated earlier (8). As shown in Table I, HOXB13 regulated the expression of multiple genes involved in cell motility and adhesion. The most notable gene regulated by HOXB13 was SPDEF, a SAM-pointed domain containing the Ets transcription factor. The SPDEF, also known as the PDEF (15), functions as an anti-invasive gene in prostate or breast cancer $(17,19)$.

Establishment of HOXB13 and/or PDEF stably transfected $P C 3$ prostate cancer cells. The forced expression of the PDEF was induced in both wild-type PC3 and PC3-HOXB13 cells. Fig. 3A shows its expression, and Fig. 3B the densitometric evaluation. The expression of HOXB13 alone significantly reduced PDEF expression $(0.34 \pm 0.05$ vs $0.17 \pm 0.04)(\mathrm{p}<0.05)$, and the expression of both HOXB13 and PDEF reduced the expression of PDEF $(0.40 \pm 0.02)$ in comparison to the PDEF alone $(0.51 \pm 0.06)(p<0.05)$, suggesting that the reduced expression of the PDEF in HOXB13 and PDEF-overexpressed PC3 cells derived from the suppression of the endogenous PDEF by HOXB13.

HOXB13 counteracts the PDEF-mediated inhibition of prostate cancer cell invasion. To determine whether the regulation 
A
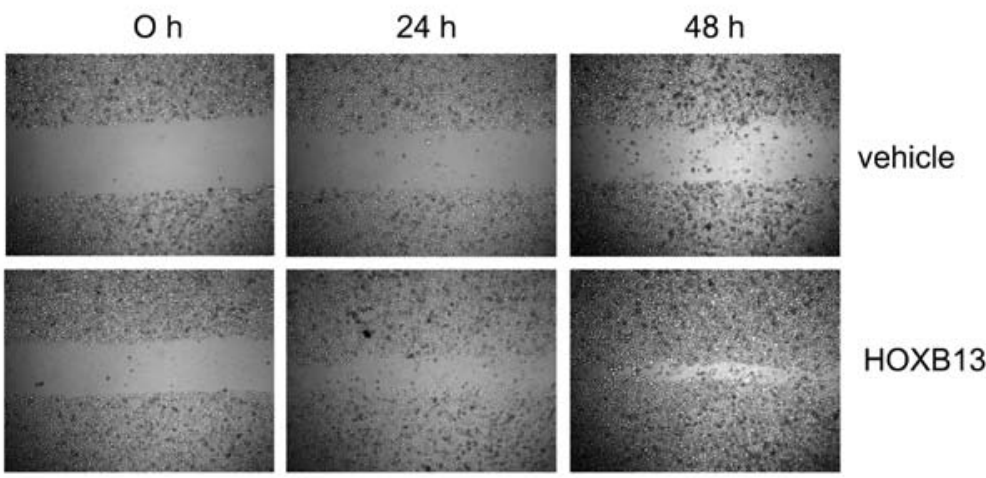

B
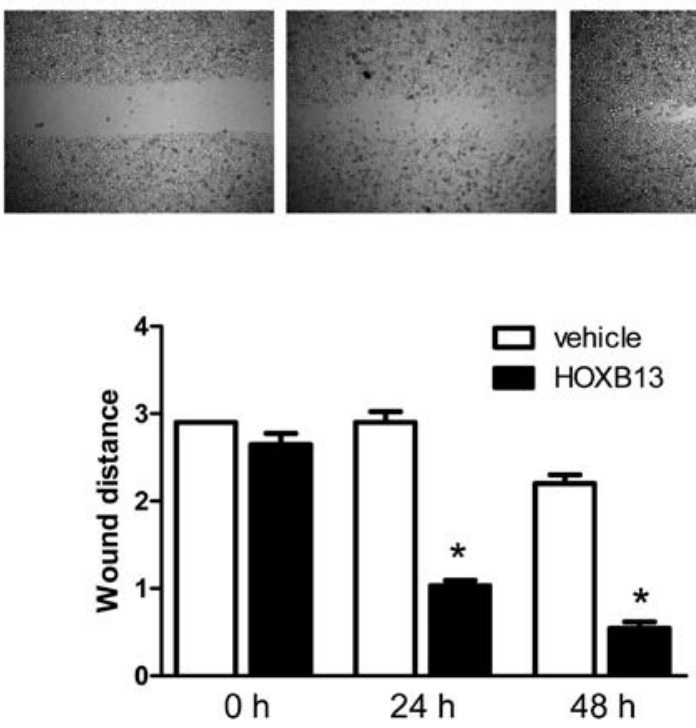

Figure 2. HOXB13 promoted the migration potential of PC3 prostate cancer cells. (A) Cells were plated onto 60-mm dishes, scratched with pipette tips, and photographed at 24 and $48 \mathrm{~h}$ by phase-contrast microscopy. (B) Five randomly selected points along each wound were marked, and the horizontal distance of migrating cells from the initial wound was measured. Presented data are from at least three independent experiments performed in duplicate. ${ }^{*}$ Student's $t$-test $(\mathrm{p}<0.05)$.

A

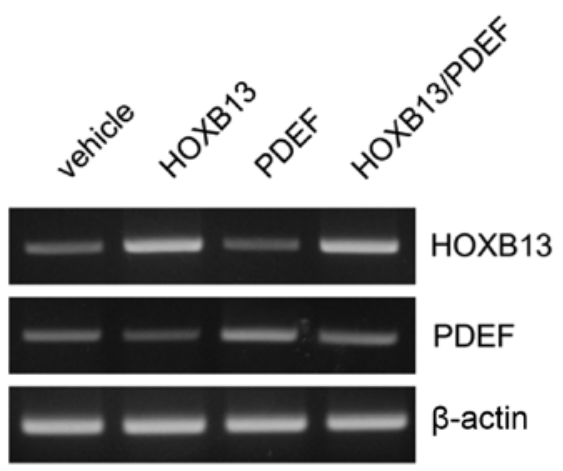

B

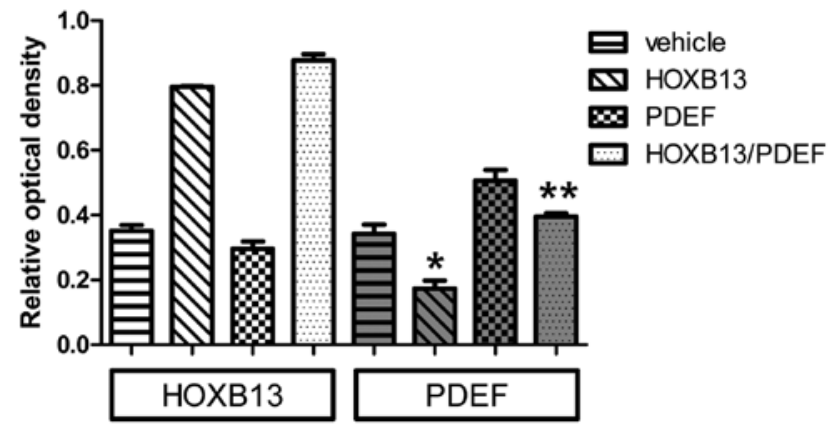

Figure 3. Establishment of HOXB13 and/or the PDEF stably transfected PC3 prostate cancer cells. (A) Stably transfectd PC3 cells expressing HOXB13, the PDEF, or both, were analyzed by RT-PCR. (B) The expression of RNA was measured by densitometer. ${ }^{* * *}$ Student's t-test $(\mathrm{p}<0.05)$. of PDEF expression would influence the invasive potential of HOXB13, HOXB13- and/or PDEF-overexpressed PC3 cells were applied to a transwell assay. Fig. 4A shows the invaded cells, and Fig. 4B shows them as a bar graph after the cells were counted on randomly selected multiple fields. HOXB13 stimulated Matrigel-coated transwell migration $(116.8 \pm 13.92$ vs $249.8 \pm 9.23)(\mathrm{p}<0.05)$, whereas the PDEF reduced invaded cells $(116.8 \pm 13.92$ vs $83.8 \pm 7.89)(\mathrm{p}<0.05)$ (Fig. 4B). The dual expression of HOXB13 and the PDEF reduced the number of cells $(175.6 \pm 10.36)$ relative to HOXB13 alone $(249.8 \pm 9.23)$ but increased it relative to the PDEF alone $(83.8 \pm 7.89)(\mathrm{p}<0.05)$. The co-overexpression of HOXB13 and the PDEF inhibited cell migration by $\sim 30 \%$ relative to HOXB13 alone and stimulated it by $\sim 50 \%$ relative to the PDEF alone. The MTT in vitro proliferation assay showed no growth difference up to $36 \mathrm{~h}$ of incubation between four different cell types (Fig. 4C). These results indicate that HOXB13 manipulated the PDEF-mediated inhibition of the invasive potential of PC3.

HOXB13 counteracts the expression of PDEF targets involved in cancer cell invasion. Cancer cells express a high level of matrix metalloproteinases (MMPs), which are known to facilitate the initiation, invasion and metastasis of tumors (20). In HOXB13- and PDEF-manipulated cells, the expression of PDEF target proteins was evaluated, including MMP-9 (16) and survivin (19). MMPs represent a family of enzymes whose function is related mainly to the degradation of extracellular matrix proteins and are necessary for cell invasion. In addition, 
Table I. List of HOXB13 target genes involved in cancer cell migration and invasion.

\begin{tabular}{|c|c|c|c|}
\hline Gene & Fold change & Accession no. & Description \\
\hline \multicolumn{4}{|l|}{ Cell motility } \\
\hline CRYAB & -4.88 & NM_001885 & Crystallin, $\alpha$ B \\
\hline KCNMA1 & -3.28 & NM_002247 & Potassium large conductance calcium-activated channel, subfamily $\mathrm{M}, \alpha 1$ \\
\hline HMMR & 4.14 & NM_012485 & Hyaluronan-mediated motility receptor (RHAMM) \\
\hline TNFRSF12A & -3.06 & NM_016639 & Tumor necrosis factor receptor superfamily, member $12 \mathrm{~A}$ \\
\hline MYLIP & 3.29 & NM_013262 & Myosin regulatory light chain interacting protein \\
\hline CKLFSF6 & 3.07 & NM_017801 & Chemokine-like factor super family 6 \\
\hline LAMA3 & -4.11 & NM_198129 & Laminin, $\alpha 3$ \\
\hline LAMA1 & -6.07 & NM_005559 & Laminin, $\alpha 1$ \\
\hline MMP2 & 2.13 & NM_004530 & Matrix metalloproteinase 2 \\
\hline MMP16 & 7.77 & NM_022564 & Matrix metalloproteinase 16 \\
\hline MMP27 & 3.78 & NM_022122 & Matrix metalloproteinase 27 \\
\hline SERPINI1 & 17.76 & NM_005025 & Serine proteinase inhibitor, clade I, member 1 \\
\hline SERPINC1 & 5.71 & NM_000488 & Serine proteinase inhibitor, clade $\mathrm{C}$, member 1 \\
\hline SPDEF & -12.38 & NM_012391 & SAM pointed domain containing ets transcription factor \\
\hline OCLN & 10.84 & NM_002538 & Occludin \\
\hline \multicolumn{4}{|l|}{ Cell adhesion } \\
\hline STIM2 & 5.28 & NM_020860 & Stromal interaction molecule 2 \\
\hline DSG4 & 12.72 & NM_177986 & Desmoglein 4 \\
\hline PKD2 & 4.86 & NM_000297 & Polycystic kidney disease 2 \\
\hline CD47 & 4.05 & NM_198793 & CD47 antigen \\
\hline CDA08 & 3.35 & NM_030790 & T-cell immunomodulatory protein \\
\hline PPFIA2 & 3.65 & NM_003625 & Protein tyrosine phosphatase receptor type $\mathrm{f}$ (PTPRF), interacting protein, $\alpha 2$ \\
\hline PPFIBP1 & -4.93 & NM_177444 & PTPRF interacting protein, binding protein 1 \\
\hline RHOB & -4.51 & NM_004040 & Ras homolog gene family, member B \\
\hline DST & 3.2 & NM_183380 & Destrin \\
\hline TNFRSF12A & -3.06 & NM_016639 & Tumor necrosis factor receptor superfamily, member $12 \mathrm{~A}$ \\
\hline LRIG3 & 3.99 & NM_153377 & Leucine-rich repeats and immunoglobulin-like domains 3 \\
\hline NRXN1 & 3.33 & NM_138735 & Neurexin 1 \\
\hline
\end{tabular}

A

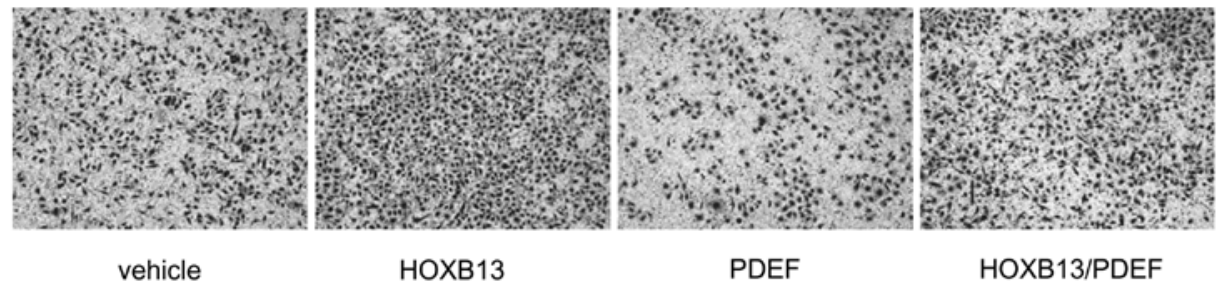

B
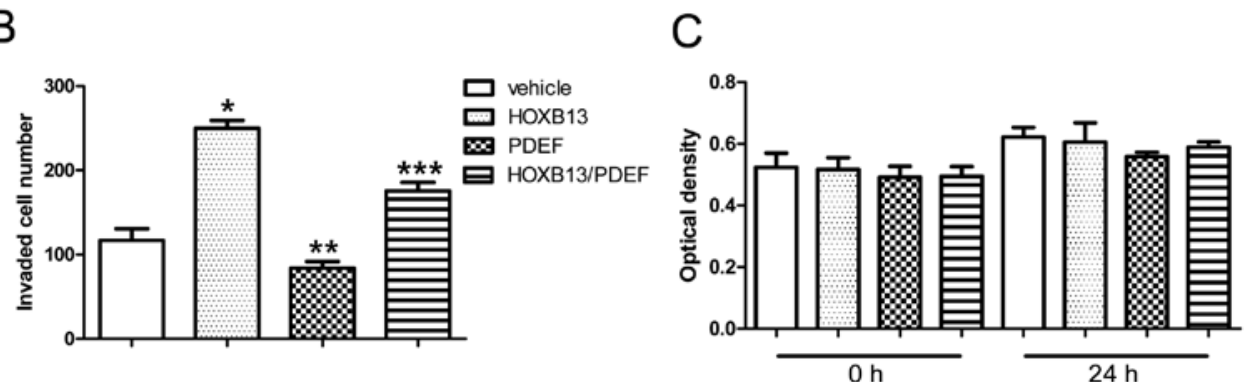

Figure 4. HOXB13 counteracted the PDEF-mediated inhibition of prostate cancer cell invasion. (A) The 24-well transwell chambers were covered with $1 \%$ gelatin solution to the upper chamber and cells were incubated in the chamber overnight at $37^{\circ} \mathrm{C}$ in the $\mathrm{CO}_{2}$ incubator. After incubation, gelatin solution was aspirated and the upper chamber was dried for $>2 \mathrm{~h}$. Media $(500 \mu \mathrm{l})$ containing fibronectin $(20 \mu \mathrm{g} / \mathrm{ml})$ were added to the bottom chamber wells. Cells were seeded in a serum-free medium into the upper chamber and incubated for $24 \mathrm{~h}$ in the $\mathrm{CO}_{2}$ incubator. After $24 \mathrm{~h}$ of incubation, the cells were stained by DiffQuick and analyzed by microscope. (B) Cells were counted and shown as a bar graph. *,**,***Student's t-test (p<0.05). (C) MTT in vitro proliferation assay was performed at $8 \mathrm{~h}$ after cells were seeded $(0 \mathrm{~h})$ and at $24 \mathrm{~h}$ after first assay $(24 \mathrm{~h})$. 


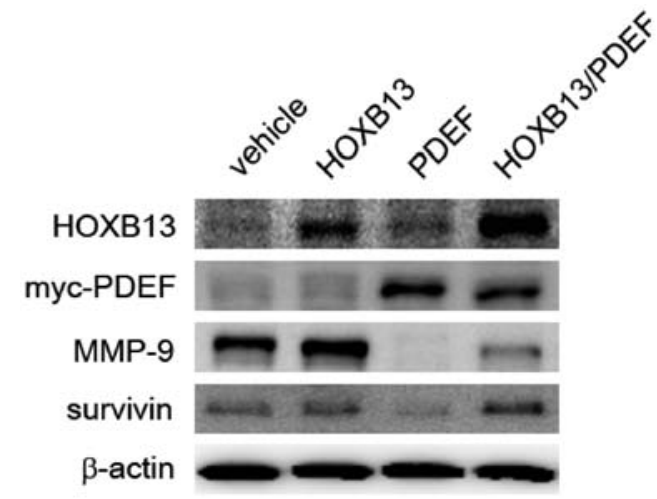

Figure 5. HOXB13 counteracted the expression of PDEF targets involved in cancer cell invasion. Cells were grown in 5\% FBS-RPMI media and then lysed and applied to a western blot analysis.

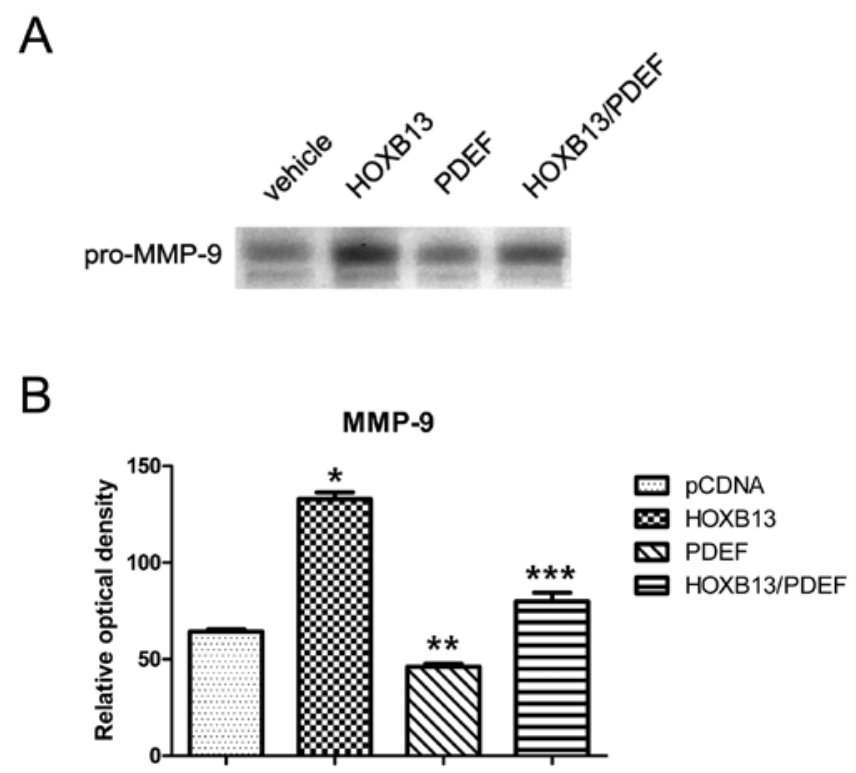

Figure 6. Gelatin zymography of MMP-9 in PC3 prostate cancer cells. Gelatin zymography was performed using conditioned media from each genetically manipulated PC3 cell line. (A) Zymography exhibited the gelatinolytic activity of MMP-9 in conditioned media. (B) Densitometric analyses of the gelatinolytic activity of MMP-9 are shown in corresponding cells. ${ }^{* * * * * * *}$ Student's t-test $(\mathrm{p}<0.05)$

increased MMP activity is associated with tumor metastasis. Survivin is an anti-apoptotic protein expressed in most forms of cancer, including prostate cancer. Survivin inhibits caspase activation, thereby facilitating the negative regulation of apoptosis, and is a significant contributor to the development of resistance to hormonal therapy in prostate cancer cells (21). As shown in Fig. 5, HOXB13 stimulated the expression of both MMP-9 and surviving, and their expression was inhibited by PDEF expression. The expression of HOXB13 partly mitigated the PDEF-mediated inhibition of MMP-9 and survivin.

HOXB13 restores matrix metalloproteinase activity suppressed by the PDEF. To determine whether the regulation of MMP-9 by HOXB13-mediated PDEF suppression would show functional activity, gelatin zymography was performed using conditioned media from each genetically manipulated PC3 cell line. A major band migrating at 92-kDa molecular mass was observed, and this conceivably corresponded to latent forms of MMP-9. Gelatin zymography exhibited the gelatinolytic activity of MMP-9 in conditioned media (Fig. 6A). The conditioned medium from untransfected PC3 cells was used as a control (lane $1 ; 64.33 \pm 1.28$ ). MMP-9 was activated by HOXB13 (lane 2; 132.87 \pm 3.46 ), but inhibited by the PDEF (lane $3 ; 46.05 \pm 1.61)$. HOXB13 partly mitigated the reduction

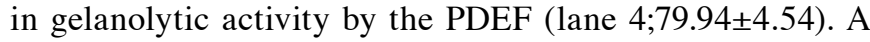
densitometric analysis of the gelatinolytic activity of MMP-9 revealed that HOXB13 significantly increased the PDEFmediated repression of MMP-9 activity ( $p<0.05)$ (Fig. 6B).

\section{Discussion}

The prostate-derived Ets factor (PDEF) is originally isolated from normal prostate tissue, and its expression has been demonstrated in other epithelial tissues, including the ovary, breast, prostate and colon (15,22-24). The PDEF has been widely investigated for its role in tumor development and progression, mostly in the case of prostate or breast cancer. However, this role has been controversial, either being a positive tumor cell promoter or a tumor suppressor in various systems under different conditions. Previous studies have shown that the PDEF generally functions as a negative regulator of cell growth, migration and invasion (17,19,22,24-26). The PDEF facilitates breast cancer growth through the negative regulation of anti-apoptotic survivin or p21 tumor suppressor expression $(19,24,27)$, and the loss of the PDEF is associated with increased MMP-9 or SLUG expression and activity in aggressive prostate cancer for the promotion of metastatic potential $(16,28)$.

This study is motivated by a research study demonstrating the role of HOXB13 in promoting prostate cancer cell invasion (10). A highly invasive PC3 prostate cancer cell line was employed for the demonstration of the role of HOXB13 in prostate cancer cell migration and invasion. The forced expression of HOXB13 inversely regulated the expression of the PDEF, resulting in the promotion of prostate cancer migration and invasion. In addition, HOXB13 negatively regulated the expression of the two most widely known PDEF target proteins, namely MMP-9 and surviving, which are involved in cancer cell metastasis and proliferation, respectively. Accordingly, the expression of HOXB13 and the PDEF in PC3 cells led to partial restoration of the expression of both PDEF target proteins and invasive potential, which were inhibited by the PDEF. This implies that the restoration of invasion by HOXB13 derived from the suppression of the endogenous PDEF by HOXB13 overexpression. HOXB13 was also involved in cancer cell metastasis. Previous studies have shown that HOXB13 facilitates the invasion of prostate, breast, and endometrial cancer cells $(3,4,29)$. It has been demonstrated that HOXB13 functions as a positive growth regulator of androgen-independent prostate cancer cell growth by modulating the RB/E2F1 signaling pathway (9).

The PDEF is also involved in the progression of prostate, breast and ovary cancer cells $(18,22,27,30,31)$. PDEF expression is widely observed in benign prostatic tissues and is downregulated or lost in prostate carcinomas. The PDEF is inversely correlated with Gleason scores, and patients with PDEF-positive tumors show significantly longer survival 
$(16,18,31)$. In ovarian cancer, positive PDEF expression in patient tumor lesions is associated with a favorable prognosis with longer overall survival. The forced expression of the PDEF in PDEF-negative ovarian tumor cells has been shown to inhibit tumor cell growth, induce apoptosis, downregulate survivin expression and reduce its promoter activity (30). The PDEF protein is also reduced in human invasive breast cancer and is absent in invasive breast cancer cell lines (22).

The results of this study demonstrate an inverse correlation between HOXB13 and the PDEF by the mechanism underlying the targeting of PDEF expression by HOXB13. In normal tissues, the expression pattern of HOXB13 has been found to be similar to that of the PDEF, which is exclusively expressed in the prostate and colon (1). However, HOXB13 expression in the breast has been found to be lower than PDEF expression (29). Both the PDEF and HOXB13 are expressed mainly in the nucleus of prostatic epithelial cells. PDEF expression appears to be inversely correlated with prostate cancer malignancy, and the alteration of HOXB13 expression during prostate cancer development is not clearly observed, presumably because of the multifocal nature of prostate cancer. Several studies have suggested that HOXB13 expression changes during prostate cancer development and malignant progression (5,9,32-34). HOXB13 expression is correlated with the Gleason score (33) and is overexpressed in castration-resistant prostate cancer cells relative to androgen-dependent cancer cells (9). The alteration of HOXB13 expression has also been observed in tumors from non-prostate cells. The expression of HOXB13 is downregulated in colon, skin and kidney tumors (35-39). On the other hand, the overexpression of HOXB13 has been observed in breast, ovary and uterine endometrium tumors (2-4). These mixed results suggest that the role of HOXB13 in cancer cell growth depends on the tissue and cellular status. For example, the forced expression of HOXB13 negatively regulates the growth of prostate cancer cells through the regulation of androgen receptor-mediated signaling or $\beta$-catenin and TCF-mediated signaling (6-8). In the context of an androgen-free environment, HOXB13 functions as a growth stimulator, as in the case of castration-resistant prostate cancer cells. The latter function appears to be mediated through the regulation of multiple signaling pathways, including $\mathrm{RB} / \mathrm{E} 2 \mathrm{~F}$ (9) and zinc/NF- $\mathrm{B}$ signals (unpublished data).

In this study, the results demonstrate that HOXB13 promoted the invasive potential of PC3 prostate cancer cells through the negative regulation of the PDEF and thus that HOXB13 counteracted the expression of PDEF target genes involved in the invasion of prostate cancer cells, namely MMP-9. Because of the lack of information on the PDEF promoter, it remains unclear how HOXB13 suppresses PDEF expression, although this should broaden knowledge of the HOXB13-mediated promotion of prostate cancer malignancy. Given that very little information is available on the regulation of PDEF expression and on the pattern of mutual HOXB13 and PDEF expression, the results of this study are expected to draw more research attention to this field.

\section{Acknowledgements}

This study was supported by a grant from the National Research Foundation (NRF) of Korea (MRC, 2011-0030132) funded by the Korea government, by a Basic Science Research Program grant (2010-0003838) from the NRF of Korea, and by a grant from the Chonnam National University Hospital Research Institute of Clinical Medicine (CRI 120061-31).

\section{References}

1. Sreenath T, Orosz A, Fujita K and Bieberich CJ: Androgenindependent expression of hoxb-13 in the mouse prostate. Prostate 41: 203-207, 1999.

2. Ma XJ, Wang Z, Ryan PD, et al: A two-gene expression ratio predicts clinical outcome in breast cancer patients treated with tamoxifen. Cancer Cell 5: 607-616, 2004.

3. Miao J, Wang Z, Provencher H, et al: HOXB13 promotes ovarian cancer progression. Proc Natl Acad Sci USA 104: 17093-17098, 2007.

4. Zhao Y, Yamashita T and Ishikawa M: Regulation of tumor invasion by HOXB13 gene overexpressed in human endometrial cancer. Oncol Rep 13: 721-726, 2005.

5. Edwards S, Campbell C, Flohr P, et al: Expression analysis onto microarrays of randomly selected cDNA clones highlights HOXB13 as a marker of human prostate cancer. Br J Cancer 92: 376-381, 2005.

6. Jung C, Kim RS, Lee SJ, Wang C and Jeng MH: HOXB13 homeodomain protein suppresses the growth of prostate cancer cells by the negative regulation of T-cell factor 4. Cancer Res 64: 3046-3051, 2004.

7. Jung C, Kim RS, Zhang HJ, Lee SJ and Jeng MH: HOXB13 induces growth suppression of prostate cancer cells as a repressor of hormone-activated androgen receptor signaling. Cancer Res 64: 9185-9192, 2004.

8. Kim SD, Park RY, Kim YR, et al: HOXB13 is co-localized with androgen receptor to suppress androgen-stimulated prostatespecific antigen expression. Anat Cell Biol 43: 284-293, 2010.

9. Kim YR, Oh KJ, Park RY, et al: HOXB13 promotes androgen independent growth of $\mathrm{LNCaP}$ prostate cancer cells by the activation of E2F signaling. Mol Cancer 9: 124, 2010.

10. Kim YR, Kim IJ, Kang TW, et al: HOXB13 downregulates intracellular zinc and increases NF-kappaB signaling to promote prostate cancer metastasis. Oncogene: 7 Oct, 2013 (Epub ahead of print). doi:10.1038/onc.2013.404 2013.

11. Sharrocks AD, Brown AL, Ling Y and Yates PR: The ETS-domain transcription factor family. Int J Biochem Cell Biol 29: 1371-1387, 1997.

12. Clark JP and Cooper CS: ETS gene fusions in prostate cancer. Nat Rev Urol 6: 429-439, 2009.

13. Kumar-Sinha C, Tomlins SA and Chinnaiyan AM: Recurrent gene fusions in prostate cancer. Nat Rev Cancer 8: 497-511, 2008.

14. Tomlins SA, Bjartell A, Chinnaiyan AM, et al: ETS gene fusions in prostate cancer: from discovery to daily clinical practice. Eur Urol 56: 275-286, 2009.

15. Oettgen P, Finger E, Sun Z, et al: PDEF, a novel prostate epithelium-specific ets transcription factor, interacts with the androgen receptor and activates prostate-specific antigen gene expression. J Biol Chem 275: 1216-1225, 2000.

16. Johnson TR, Koul S, Kumar B, et al: Loss of PDEF, a prostatederived Ets factor is associated with aggressive phenotype of prostate cancer: regulation of MMP 9 by PDEF. Mol Cancer 9: $148,2010$.

17. Gu X, Zerbini LF, Otu HH, et al: Reduced PDEF expression increases invasion and expression of mesenchymal genes in prostate cancer cells. Cancer Res 67: 4219-4226, 2007.

18. Turner DP, Findlay VJ, Moussa O, et al: Mechanisms and functional consequences of PDEF protein expression loss during prostate cancer progression. Prostate 71: 1723-1735, 2011.

19. Ghadersohi A, Pan D, Fayazi Z, Hicks DG, Winston JS and Li F: Prostate-derived Ets transcription factor (PDEF) downregulates survivin expression and inhibits breast cancer cell growth in vitro and xenograft tumor formation in vivo. Breast Cancer Res Treat 102: 19-30, 2007.

20. Lynch CC and Matrisian LM: Matrix metalloproteinases in tumor-host cell communication. Differentiation 70: 561-573, 2002.

21. Zhang M, Latham DE, Delaney MA and Chakravarti A: Survivin mediates resistance to antiandrogen therapy in prostate cancer. Oncogene 24: 2474-2482, 2005. 
22. Feldman RJ, Sementchenko VI, Gayed M, Fraig MM and Watson DK: PDEF expression in human breast cancer is correlated with invasive potential and altered gene expression. Cancer Res 63: 4626-4631, 2003.

23. Feldman RJ, Sementchenko VI and Watson DK: The epithelialspecific Ets factors occupy a unique position in defining epithelial proliferation, differentiation and carcinogenesis. Anticancer Res 23: 2125-2131, 2003

24. Moussa O, Turner DP, Feldman RJ, et al: PDEF is a negative regulator of colon cancer cell growth and migration. J Cell Biochem 108: 1389-1398, 2009.

25. Turner DP, Findlay VJ, Kirven AD, Moussa O and Watson DK: Global gene expression analysis identifies PDEF transcriptional networks regulating cell migration during cancer progression. Mol Biol Cell 19: 3745-3757, 2008.

26. Turner DP, Moussa O, Sauane M, Fisher PB and Watson DK Prostate-derived ETS factor is a mediator of metastatic potential through the inhibition of migration and invasion in breast cancer. Cancer Res 67: 1618-1625, 2007.

27. Schaefer JS, Sabherwal Y, Shi HY, et al: Transcriptional regulation of p21/CIP1 cell cycle inhibitor by PDEF controls cell proliferation and mammary tumor progression. J Biol Chem 285 11258-11269, 2010.

28. Findlay VJ, Turner DP, Yordy JS, et al: Prostate-derived ETS factor regulates epithelial-to-mesenchymal transition through both SLUG-dependent and independent mechanisms. Genes Cancer 2: 120-129, 2011.

29. Ma XJ, Hilsenbeck SG, Wang W, et al: The HOXB13:IL17BR expression index is a prognostic factor in early-stage breast cancer. J Clin Oncol 24: 4611-4619, 2006.

30. Ghadersohi A, Odunsi K, Zhang S, et al: Prostate-derived Ets transcription factor as a favorable prognostic marker in ovarian cancer patients. Int J Cancer 123: 1376-1384, 2008.
31. Ghadersohi A, Sharma S, Zhang S, et al: Prostate-derived Ets transcription factor (PDEF) is a potential prognostic marker in patients with prostate cancer. Prostate 71: 1178-1188, 2011.

32. Fromont G, Chene L, Latil A, et al: Molecular profiling of benign prostatic hyperplasia using a large scale real-time reverse transcriptase-polymerase chain reaction approach. J Urol 172: 1382-1385, 2004

33. Jeong TO, Oh KJ, Xuan Nguyen NT, et al: Evaluation of HOXB13 as a molecular marker of recurrent prostate cancer. Mol Med Rep 5: 901-904, 2012.

34. Johnson PH, Walker RP, Jones SW, et al: Multiplex gene expression analysis for high-throughput drug discovery: screening and analysis of compounds affecting genes overexpressed in cancer cells. Mol Cancer Ther 1: 1293-1304, 2002.

35. Birkenkamp-Demtroder K, Olesen SH, Sorensen FB, et al: Differential gene expression in colon cancer of the caecum versus the sigmoid and rectosigmoid. Gut 54: 374-384, 2005.

36. Hoek K, Rimm DL, Williams KR, et al: Expression profiling reveals novel pathways in the transformation of melanocytes to melanomas. Cancer Res 64: 5270-5282, 2004.

37. Jung C, Kim RS, Zhang H, et al: HOXB13 is downregulated in colorectal cancer to confer TCF4-mediated transactivation. Br J Cancer 92: 2233-2239, 2005.

38. Komuves LG, Ma XK, Stelnicki E, Rozenfeld S, Oda Y and Largman C: HOXB13 homeodomain protein is cytoplasmic throughout fetal skin development. Dev Dyn 227: 192-202, 2003.

39. Okuda $\mathrm{H}$, Toyota $\mathrm{M}$, Ishida $\mathrm{W}$, et al: Epigenetic inactivation of the candidate tumor suppressor gene HOXB13 in human renal cell carcinoma. Oncogene 25: 1733-1742, 2006. 